\title{
EDUCAR E INVESTIGAR, CARA Y SELLO DEL MISMO PROCESO ${ }^{1}$
}

Educate and investigate, face and seal of the same process

Educar e investigar, cabeças e caudas do mesmo proceso

\author{
Carlos Calvo Muñoz \\ Universidad de La Serena, Chile. Fono: +56 98360 2544. Correo electrónico: \\ carlosmcalvom@gmail.com
}

\begin{abstract}
Resumen
Es este artículo planteamos que la educación que tiene lugar en las escuelas no es la misma educación que fluye naturalmente en los seres humanos y que la observamos nítidamente en la primera infancia y que continua toda la vida. En atención a ello, señalamos que la educación consiste en un proceso de creación de relaciones que puede ir de lo posible a lo probable y a lo realizable; mientras que la escolarización se trata de un proceso de repetición de relaciones pre-establecidas. Dado lo anterior, educar e investigar constituyen expresiones del mismo proceso, mientras que en la escuela coexisten en ámbitos separados. Finalmente afirmamos que educar es asombrar con un misterio, en tanto que investigar consiste en intentar develar ese misterio.
\end{abstract}

Palabras clave: educación, escolarización, investigación, relaciones.

\begin{abstract}
The purpose of this article is to state that the education that takes place in the schools is not the same that occurs naturally among human beings, which is clearly observed in early childhood and continues throughout life. Considering that, we can say that education consists of a process of creating possible relations which moves from the possible to the probable and from there to the achievable, whereas schooling is about a
\end{abstract}

\footnotetext{
1 Algunas de las ideas desarrolladas en este articulo han sido trabajadas en "La formación del educador investigador" (Calvo 2002) y han sido complementadas con las ideas emergentes del Proyecto FONDECYT 1110577 (2011-2014): “Asombros educativos informales y propensión a aprender”.
} 
process of repetition of preset relations. Therefore, education and research represent expressions of the same process. In the school, however, both concepts coexist in separate environments. Finally, we affirm that educating is to wonder with a mystery, while researching consists of attempting to unveil that mystery.

Key words: education, schooling, investigation, relations.

\section{Resumo}

Neste artigo propomos que a educação escolar não é a mesma educação que flui naturalmente nos seres humanos e que observamos, nitidamente, na primeira infância e ao longo da vida. Destacando isso, afirmamos que a educação consiste em um processo de criação de relações que podem ir do possível ao provável e ao realizável, enquanto a escolarização se apresenta como um processo de repetição de relações préestabelecidas. Logo, educar e investigar constituem-se como expressões do mesmo processo, enquanto que na escola coexistem em âmbitos separados. Finalmente, afirmamos que educar é assombrar-se com um mistério, portanto, investigar consiste em tentar desvendar esse mistério.

Palavras-chave: educação, escolarização, investigação, relações.

\section{La aventura de educar e investigar}

Dado que quien educa asombra con algún misterio y que quien investiga intenta develar dicho misterio, ambas acciones no se pueden separar ni menos oponerlas antagónicamente: o educo o investigo; por el contrario, entre ellas existe una relación de continua recursividad, gracias a la cual educar e investigar conforman una trama compleja donde se retroalimentan mutuamente. Se trata de una "causalidad circular de naturaleza recursiva o retroactiva que desafía las nociones de causa / efecto de la ciencia tradicional” (Moraes, 2015:35), que son las nociones con las cuales muchos centros formadores sustentan la formación de los educadores.

Al educar se provoca al educando para que inicie un proceso que le permita relacionar creativamente aquello que le provoca. Procede sin orden preestablecido mientras está abierto a lo que puede acontecer, sin importar si sucederá o no. Es 
totalmente lúdico y placentero. Al investigar la creación se entreteje con la observación que permite descubrir regularidades que pueden conformar patrones complejos que fluyen caóticamente desde lo posible hacia lo probable y que tal vez podría ser realizable si se cumplen ciertas condiciones. La ayuda del educador mediador es crucial en este proceso, pues ayuda a observar y seleccionar criterios orientadores y provisorios.

Al educar e investigar se avanza y retrocede con distintos ritmos e intensidades entre comprensiones y confusiones, conversaciones y soliloquios, rigor metódico y distracción, en pos de la intuición serendípica que de la pista de como y por donde continuar.

Einstein utilizaba mucho la alteración de sus estados de consciencia para resolver problemas de física. Tenía un diván en su estudio; se tendía en él y tenia una bola de metal en la mano y debajo un orinal, también de metal. Mientras se estaba quedando dormido, en la fase que hay entre la vigilia y el sueño entraba en un estado alterado de consciencia, esto nos pasa a todos cuando nos vamos a dormir). Entonces la bola caía en el orinal y le despertaba, y volvía a repetirlo. Einstein contaba que los problemas más complejos los había resuelto así, en ese estado alterado de consciencia, de semi sueño. El se 'drogaba' con la dimetiltriptamina de su propia glándula pineal. Es probable que la dimetiltriptamina sea también la que genera los contenidos de nuestros sueños." (Atwell, 2015: 317)

Educar e investigar son aventura pura, desafiante e inspiradora. Una condición primordial de toda aventura es que quienes participan en ella no pueden tener certeza de cómo fluirá el proceso ni mucho menos de cuál será el desenlace final, aunque puedan suponer algunos resultados con distintos grados de certeza.

Es inconcebible una aventura que no sea caótica y que sus procesos no generen emergencias, bifurcaciones y recursividades diversas y complejas. Al investigador no le queda más que estar atento, especialmente al detalle ínfimo, que parece irrelevante, pero que puede ejercer la influencia sutil, equivalente al aleteo de la mariposa que altera el curso de los acontecimientos (Calvo, 2016, 2005; Gleick, 1988). 
Es posible que de este hecho provenga la obsesión compulsiva de muchos administradores escolares que desean controlar todos los aspectos de los procesos de enseñanza y de aprendizaje y la tentación coercitiva por controlar permanentemente esos procesos imponiendo normas restrictivas que en vez de favorecer la enseñanza y los aprendizajes están desprofesionalizando al profesorado y estrangulando la creatividad de profesores y estudiantes.

También se teme el hecho que todos los actores de los procesos de enseñanza y de aprendizaje sean subjetivos y no halla manera de volverlos objetivos como si fueran una roca. Las pruebas estandarizadas, funcionales para el poco tiempo que tienen los profesores para escuchar con calma cuáles han sido los descubrimientos que han hecho sus alumnos, las relaciones inéditas que han creado y las reflexiones que los animan para continuar, se vuelven atractivas porque todos son evaluados, calificados, medidos del mismo modo, arguyendo objetividad, pero ocultando las injusticias que se comenten en su nombre y la superficialidad del procedimiento al excluir a la incertidumbre y la paradoja para dar lugar a la certeza, la coherencia y simetría.

La subjetividad complejiza la aventura de educar y enseñar porque en el proceso los efectos devienen causas que generan nuevos efectos que se transformarán en germen de otras secuelas a lo largo de un complejísimo bucle recursivo. Esto marea a quien se mueve en el paradigma cartesiano y es una de las principales razones por la que no cualquier persona puede enseñar e investigar en educación.

[El] bucle autogenerador o recursivo, es decir, el bucle en el que los efectos y los productos se convierten en algo necesario para la producción y para la causa de aquello que los causa y los produce. Un ejemplo evidente de ese tipo de bucle somos nosotros mismos, productos de un ciclo de reproducción biológica del cual nos convertimos, para que el ciclo continúe, en productores. Somos productos productores. Asimismo, la sociedad es el producto de las interacciones entre individuos; pero a nivel global emergen cualidades nuevas que al retroactuar sobre los individuos -el lenguaje, la cultura- les permiten realizarse como individuos. Los individuos producen la sociedad y ésta a su vez produce los individuos. De aquí podemos extraer dos consecuencias importantes. Una, de carácter lógico, es que un producto productor es incompatible con la lógica clásica. La otra es que aparece la 
noción de autoproducción y de autoorganización.” (Morin, 2016: 98).

\section{Cegueras investigativas}

Taleb en El cisne negro nos advierte sobre "nuestra ceguera respecto a lo aleatorio, en particular las grandes desviaciones" (2009:25) que intervienen en todos los procesos generando bifurcaciones que debemos atender y que, según cuál sea la elección, originará un curso de acción u otro, sin vuelta atrás porque no existe la reversibilidad del tiempo. Aunque no nos debería sorprender, igual nos deja pasmados conocer la poca fiabilidad de las predicciones en algunas áreas disciplinarias:

"De casi un millón de artículos publicados sobre política, finanzas y economía, sólo ha habido un reducido número de comprobaciones sobre la cualidad predictiva de este tipo de conocimientos. [...] después de investigar 2000 predicciones de analistas de inversiones, se demostró que no 'predecían nada' [...] "los analistas predicen peor al tiempo que tienen más fe en sus propias destreza" (Taleb, 2009:222-223)

Algunos achacarán estas deficiencias a la metodología poco fiable empleada por esos investigadores. Es probable que así sea, sin embargo, los errores en otros campos son también frecuentes. El disparate ocurrido con la construcción del puente Cau-Cau en Valdivia, Chile, es un desacierto ingenieril; también podríamos mencionar graves equivocaciones en el campo de la medicina, pero no lo haremos ya que lo que nos interesa es otro asunto.

En el mundo académico es común escuchar afirmaciones en torno a que investigar exige dedicación total y que se requiere seguir criterios exigentes y rigurosos por lo que no se puede ser buen investigador si se realizan otras actividades absorbentes, tales como la docencia. Contar con investigadores de tiempo completo que investiguen y publiquen en revistas de corriente principal es casi un imperativo universitario y de las empresas de punta. Si no formamos parte del grupo disciplinario no comprenderemos sus procedimientos ni resultados, no solo por su complejidad, especialización y vocabulario excluyente, sino también porque muchas se publican en revistas de pago muy caras y otras investigaciones tienen derechos reservados, es decir, son propiedad privada, esto es, excluyente. 
Esta situación presenta profundos problemas éticos y políticos que afectan a la sociedad mundial y, por supuesto, a los mismos científicos que se encuentran con las manos atadas porque no pueden siquiera conversar sobre lo que hacen porque deben firmar contratos que les impiden hacerlo. La mayoría de esa investigación, mucha de la cual se realiza con fondos públicos, termina en manos de las empresas privadas o de la industria militar provocando la ira e indignación de académicos, administrativos universitarios y público informado. Robert Laughlin, premio nobel de física en 1998, no advierte que

“el conocimiento más valioso en términos económicos es propiedad privada y es secreto. Los dueños de ese conocimiento no quieren hacerlo público $\mathrm{y}$, ciertamente, no quieren que el Estado le de dinero a nadie para que lo 'descubra'.... En la práctica, el derecho a aprender es de acceso restringido.” (Laughlin, 2010:7).

Por su parte, el rector de la Universidad de Harvard ha criticado a Elsevier, Springer and Wiley, que publican las revistas de alto impacto en el campo de la ciencia, por el cobro desmedido que aplican a quienes quieren -o deben- publicar en esas revistas. Acusa que sus académicos evalúan gratis para ellas, pero que cuando envían un manuscrito para que se considere su publicación deben pagar y que para leer esas revistas deben cancelar unos derechos abusivos, los que según la Biblioteca Central de la misma universidad alcanza a 3,5 millones de dólares anuales. Esta situación ha llevado a que más de 10.000 académicos de diversas instituciones hayan iniciado un boicot a Elsevier (The Guardian, 2012) y que cada día más investigadores publican en revistas de acceso abierto.

Esta situación no debe empañar los aportes de la investigación positivista, algunos de cuyos frutos lo vemos a diario en el uso de tecnología sofisticada como los smarthphones que la mayoría usamos a diario y que han entrado en las escuelas y en nuestras vidas para no salir hasta que otro producto tecnológico, insospechado por el momento por que la ciencia todavía no ha descubierto aquello que una tecnología no creada por el mismo motivo, lo sustituya por otro nuevo, tal como pasó cuando irrumpe sorpresivamente el smarthphone, con Internet, las redes sociales y las nuevas maneras de comunicarnos virtualmente, las que han impacto en la educación y la escuela 
(Brockman, 2010, Kaku, 2012, 2014, Punset, 2004).

\section{Educación y escolarización}

Para entender las complejas implicancias de lo precedente es imperativo diferenciar categóricamente los procesos educativos de los escolarizados. En algún momento, que puede ser imperceptible para el mismo niño y niña, la curiosidad les lleva a dar un paso extraordinario cuando, al considerar todo lo que ha imaginado como posible, pondere cuáles de todas esas posibilidades tienen alguna probabilidad de suceder. Entremedio de confusiones descubrirá que lo posible tal vez jamás acontezca, pero que lo probable es aquello que tiene chance de ocurrir, que es viable o factible bajo ciertas condiciones que tratará de identificar y establecer como causas o efectos; al continuar trabajando sobre aquello la idea podría ser realizable, solo si continua dedicándole atención y esfuerzo, si bien, jamás contará con la certeza de que lo logrará, pues la incertidumbre siempre será omnipresente e inevitable. (Calvo 2015: 26)

Por el contrario, en la escolarización la aventura del aprendizaje queda entrampada en la formalidad del proceso. La mayor gravedad reside en que los educandos conocen de antemano el resultado y deben aprenderlo de esa manera, lo comprendan o no. Es verdad que muchísimos profesores dirán que no es así y que se esfuerzan en que el estudiante sean el constructor de su propio aprendizaje, sin embargo, los hechos desmienten que esto suceda en la mayoría de los casos y las excepciones solo confirman la tendencia mayoritaria que se traduce en el fracaso escolar. Este fracaso no es el resultado de un enfoque o propuesta pedagógica, sino que responde al paradigma que da sentido y sostiene el modelo escolar vigente centrado en la respuesta y no en la búsqueda, la incertidumbre, la aventura.

\section{4. ¿Qué investigadores necesitamos?}

Toda investigación es bienvenida en el campo educacional y escolar a condición de que sea rigurosa y sistemática; empero no es suficiente que sea investigación de punta y altamente sofisticada para que sea útil en el trabajo diario del profesorado, pues responden a otros intereses académicos y, por que no señalarlo, dependencia financiera. 
Una consecuencia es que se pesquisan temas que no son de interés y aplicación directa en la enseñanza y el aprendizaje, los que deben ser tamizadas por la experiencia docente y las condiciones socio culturales de cada grupo.

Con frecuencia los aportes de la investigación de punta, por ejemplo, en neurociencia deben ser descodificados atendiendo al impacto que ejerce en ellos la cotidianeidad de los procesos de enseñanza y de aprendizaje en la subjetividad de los diferentes actores. Si esto no se hace se corren serios riesgos de errar en lo que podría haberse convertido en un aporte significativo y medular, tal como ha sucedido cuando se imponen modas que no se sustentan en las prácticas educativas.

Sus consecuencias son extremadamente perniciosas pues se termina inculpando al profesorado y al alumnado por el bajo rendimiento y no a los modelos que se imponen. Es por esto que el aporte de la ciencia debe ser leído desde la práctica educativa y escolar para que se complementen sinérgicamente con el acervo de conocimientos de los profesores investigadores. Un ejemplo pertinente lo encontramos en la buena etnografía educacional que aporta luces sobre como fluye el proceso educativo en la interacción educador - educando.

Necesitamos un educador investigador que educa mientras investiga o, si se quiere, que investiga mientras educa. Esto es posible porque enseñar y aprender son cualidades emergentes y recursivas que nos acompañan toda la vida, cada una transformando a la otra. "Nuestra innata habilidad para enseñar se desarrolla a lo largo del tiempo, del mismo modo como nuestra habilidad para aprender también se desarrolla a lo largo del tiempo" (Rodríguez, 2014:16).

Es importante que el campo de la educación y de la investigación educacional se orienten hacia la transdisciplina, superando el enfoque disciplinar, multidisciplinar e interdisciplinar, todos ellos sesgados por el peso de la disciplina original y ciegos ante la emergencia de complejidades inéditas.

La transdisciplinariedad no se limita a la suma o a la integración de las disciplinas para obtener un producto que trascienda. Es preciso ir un poco más allá en lo que se refiere a la manera de abordar la complejidad del fenómeno humano, 
del fenómeno de la educación, así como la complejidad de la vida." (Moraes, 2015:

92-93) [traducción personal]

\section{Formación de un educador investigador}

La formación de un educador investigador es un asunto simple y complejo, pero no superficial ni complicado. Es simple porque se trata de recuperar la inocencia, la curiosidad sin límites y el infatigable anhelo por experimentar todo que hemos gozado en nuestra infancia.

¿Quién no ha visto a un pequeño observar atentamente el ir y venir de las hormigas, al tiempo que les coloca obstáculos para saber como los salvan? Hay en su comportamiento concentración atenta a los detalles y al todo. A medida que observa convierte los datos en información al ordenarlos de acuerdo a criterios emergentes. Sin percatarse ha convertido lo simple en complejo. Cuando vuelva a jugar pondrá a prueba sus conocimientos al establecer otros parámetros de observación y experimentación, que “des-ordenarán” el orden previo. Al final, se quedará con aquel que pruebe más consistencia y coherencia, pero solo por un tiempo, hasta que comience un nuevo juego ${ }^{2}$.

La formación del educador investigador es compleja porque se trata de conjugar en una persona distintas cualidades y destrezas profesionales. Enseñar e investigar, sin bien son diferentes, tienen elementos comunes. Quien enseña asombra con el misterio, quien investiga lo hace asombrado por el misterio; quien enseña muestra regularidades, quien investiga indaga por regularidades; etc. Lo común es la apertura inocente y no ingenua ante sí mismo y el mundo ${ }^{3}$. Es inocente porque el saber no es definitivo ni la ignorancia un lastre; sería ingenuo si lo conocido le fuera suficiente y concibiera a la ignorancia un escollo.

Al educador investigador le apasiona aprender y no oculta la ignorancia porque le ayuda a comprobar la validez de sus conocimientos. No teme equivocarse, pero toma

2 Si el niño es inhibido de estas actividades lúdicas corre el riesgo de convertirse en "deprivado cultural".

3 A pesar que lo que sigue expresará situaciones extremas, aparentemente dicotómicas, no es mi intención. Al contrario, solo pretendo resaltar rasgos distintivos que no se encuentran de forma pura en nadie. 
medidas para evitar el error. Entienden que la relación que une al saber con la ignorancia es paradojal $^{4}$ y que cada nuevo aprendizaje provoca preguntas inéditas ${ }^{5}$, algunas de las cuales madurarán en hipótesis.

En su formación es tan necesaria la enseñanza sistemática y rigurosa de antecedentes epistemológicos, disciplinarios y metodológicos, como insuficiente. Es necesaria porque la ciencia es acumulativa y la docencia e investigación se llevan a cabo a partir de sus descubrimientos ${ }^{6}$. Es insuficiente porque enseñar e investigar requieren de sensibilidad y destrezas artística ${ }^{7}$ para combinar saberes, metodologías y técnicas ${ }^{8}$. $\mathrm{Ni}$ enseñar ni investigar son tareas instrumentales, sino actos creativos, al mismo tiempo, que profundamente metódicos.

El educador investigador dialogará con lo que investiga gracias a preguntas que cambian sutilmente algunas condiciones o características del tema investigado: "si esto ..., entonces aquello". No es indispensable, aunque puede ser conveniente, que las preguntas sean coherentes y convergentes con la ortodoxia científica y que estén claramente formuladas, pues con frecuencia la divergencia propicia descubrimientos imprevistos, que aumentan el deseo por comprender las relaciones que se insinúan. El entusiasmo le hará soñar con el tema, de día y de noche, favoreciendo la intuición ${ }^{9}$, que irrumpirá repentinamente disfrazada como imagen onírica o poética ${ }^{10}$. Será capaz de reconocerla si está completamente imbuido en el tema; en caso contrario, no lo hará.

El educador investigador debe saber que su trabajo será valioso a condición que

\footnotetext{
4 La relación saber - ignorancia puede graficarse como un iceberg, correspondiendo la parte superior al saber y la sumergida a la ignorancia. Por más que nieve jamás el saber superará a la ignorancia. Aunque esta imagen es buena, puede sugerir que la proporción saber / ignorancia se mantendrá constante. Esto es falso, pues la ignorancia aumenta en la medida que se aprende. Evidentemente, se trata de una ignorancia diferente a la de quien ni siquiera es conciente de ella.

5 Son inéditas porque son nuevas para el sujeto. No importa si son antiguas en la ciencia.

6 Sin duda que la docencia también se nutre del arte y otras fuentes.

7 No deja de ser una coincidencia curiosa que grandes científicos han sido músicos.

8 Esto es particularmente serio hoy en día con los softwares de apoyo a la docencia e investigación (SPSS, Atlas/Ti, etc.), cuya ayuda instrumental es valiosa solo para el que comprende el sentido de su uso.

9 La intuición no es la conclusión de razonamientos deductivos o inductivos, sino abductivos.

10 A este fenómeno le han denominado "serendipia" y tiene lugar a condición del trabajo riguroso, sistemático y exigente, tal como reconocen connotados investigadores.
} 
lo ejecute con entusiasmo, voluntad y excelencia ${ }^{11}$. Sin embargo, no puede olvidar que no todo es asombro y entusiasmo, pues también se realizan tareas rutinarias como tabular datos o repetir pruebas de laboratorio. La rutina, muchas veces tediosa, puede ocultar sorpresas interesantes para el desatento, que aparecen ocasionalmente y sin orden aparente, como si buscarán que alguien repare en ellas, a condición de escudriñarlas desde otra perspectiva conceptual y metodológica, divergente con la anterior.

Si un educador investigador es capaz de asombrarse ante lo conocido, convertirá lo ordinario en extraordinario, dejará que el objeto se muestre en plenitud, observará sin pretender explicar, develará relaciones no previstas, indagará irregularidades no sospechadas y profundizará en complejidades emergentes. Si solo le sorprende lo desconocido le será difícil trascender las fronteras epistemológicas y científicas de su disciplina, le costará entablar el diálogo interdisciplinario y, en el mejor de los casos, será un buen repetidor, pero no un innovador.

La formación de un educador investigador implica el desarrollo de la docencia y la investigación como competencias armónicas e incluyentes. Sobre este punto discrepan especialmente quienes acostumbran a sacralizar y dicotomizar actividades. Argumentan que se puede investigar sin docencia y que muchos docentes no investigan. Esto es correcto en el contexto de las limitaciones epistemológicas y funcionales de la escuela contemporánea, pero no en una escuela "des-escolarizada", que ha recuperado sus cualidades educacionales ${ }^{12}$, donde es inconcebible un docente que no investigue, pues la investigación fluye del preguntar inherente al ser humano ante lo novedoso. Entre los que no preguntan se encuentra el fanático, que cree saber todo, el reprimido,

\footnotetext{
11 Se atribuye a Einstein haber afirmado que su trabajo tenía un $1 \%$ de inspiración y un $99 \%$ de transpiración.

12 No es novedad afirmar que la escuela requiere una transformación radical, que vaya más allá de las reformas en boga, que recrean la escuela sin recuperar los procesos auténticamente educativos que tienen lugar fuera de ella. Hay que "des-escolarizar" la escuela, quitándole lo que tiene de escolar, reinventándola a partir de los procesos educativos informales y la etnoeducación.
} 
que teme hacerlo, y el escolar, que ha aprendido a responder con estereotipos ${ }^{13}$.

Dado que el investigador "perturba la naturaleza y observa si revela un secreto" (Wilson, 2014: 92), la investigación es una manera de intervenir en la naturaleza, presionándola a desenvolverse dentro de parámetros conceptuales y metodológicos específicos, lo que obliga a precisar con cuidado qué se investigará y cómo. Este imperativo exige respetar las exigencias procedimentales que garantizan que se avanzará por buen camino. Si no se cumplen, es altamente probable que los resultados sean irrelevantes, si no falsos. Respetar el procedimiento ortodoxo permite progresar en el desarrollo de la ciencia, pero solo en aquellos aspectos susceptibles de ser descubiertos desde esa perspectiva.

Para romper con la tradición se necesita divergencia creativa y mucha voluntad y convencimiento personal. No hay que olvida que grandes descubrimientos han sido posibles cuando el científico se ha atrevido a aventurarse por senderos inexplorados, lo que no le resulta fácil, no solo por las dificultades propias de la tarea, sino por los largos y estériles debates con la comunidad científica, que reacciona celosa ante lo que concibe como amenaza. Toma tiempo poder demostrar y mostrar la validez de los nuevos planteamientos $^{14}$. Lamentablemente, no habrá un solo Galileo en la ciencia, la investigación y la enseñanza ${ }^{15}$.

13 "Neruda pregunta y los niños responden" es un libro que da cuenta de algunas preguntas que Neruda se hizo y que una profesora formula a niños de 10 años. Sus respuestas asombran por la vivacidad y libertad epistemológicas que fertilizan nuevas relaciones; sin embargo, cuando 6 años después se les vuelve a preguntar, sus respuestas son estériles, predecibles y sin creatividad.

14 Llama la atención que el tiempo que requiere una idea novedosa para ser acogida por la comunidad científica sobrepasa los 15 años. Esto es mucho tiempo, especialmente si se trata de científicos que están al día en sus disciplinas y que someten a comprobación todo cuanto llega a sus manos. Al parecer, no bastan los procedimientos científicos para validar una idea, sino que se requieren de pruebas ajenas a la ciencia. Esto recuerda al astrónomo turco de El Principito que se le cree solo cuando explica la existencia del asteroride B 612 vestido con corbata.

15 En cualquier libro sobre Historia de la Educación e Historia de la Ciencia se encontrarán ejemplos de las dificultades padecidas por los innovadores para que sus ideas fuesen aceptadas por la comunidad de pares, para quienes no ha sido suficiente la evidencia acumulada. Muchas comunidades de docentes y de científicos actúan como ghettos, excluyendo y castigando a los que piensan distinto. Es conocida la dificultad para conseguir financiamiento de los investigadores que rompen con el establishment, a pesar de la calidad y consistencia de sus proyectos, que los presiona a investigar lo que promueven las Agencias, Laboratorios comerciales, Industria de la guerra, transnacionales o Bancos Internacionales, etc. 
Investigar es mucho más que definir un problema, diseñar un procedimiento e implementar la recolección e interpretación de los datos. Investigar es transformar los datos en información al atribuirle significados especiales. El que investiga descubre que no hay neutralidad ni objetividad externa independiente del sujeto, sino "objetividad consensuada" sobre criterios epistemológicos paradigmáticos comunes ${ }^{16}$. Al respecto la maestra maorí, Linda Tuhiwai nos advierte que

Desde el punto de vista del colonizado, posición desde la cual escribo y por la que opto, el término 'investigación' está intrínsecamente ligado al imperialismo y colonialismo europeos. La palabra misma, 'investigación', es quizás una de las más sucias en el vocabulario del mundo indígena. En muchos contextos indígenas, cuando se menciona esta palabra, incita silencio, conjura malos recuerdos, provoca una sonrisa que proviene del conocimiento y la desconfianza. [...] El solo hecho de saber que alguien midió nuestras 'facultades' llenando el cráneo de nuestros ancestros con semillas de mijo y que comparó dicha cantidad de semillas con la capacidad mental de pensar, ofende nuestro sentido de quiénes y qué somos.

Thompson (1985) escribe que

«Esta relativa pequeñez del cerebro se produce al descuidar del ejercicio de las facultades superiores mentales, porque a medida que se encogen los músculos por carecer de uso, es natural que, después de generaciones de indolencia mental, se disminuya el tamaño del cerebro», (Tuhiwai, 2016:19)

En consecuencia, la formación de un educador investigador va mucho más allá del aprendizaje de procedimientos metodológicos y técnicas de trabajo, que son necesarios, pero insuficientes. El meollo de la formación radica en ayudarle a desarrollar -que es una forma de despertar- la capacidad del investigador de convertir lo común y vulgar en asombroso. Si no es capaz de hacerlo, ¿cómo podrá mantener el entusiasmo en los momentos que la investigación se vuelve rutinaria? Investigar no es simulación, sino acción reflexiva y comprensiva, asombro, aburrimiento, confusión profunda que extravía, reencuentro, fascinación, etc.; en suma, es vida vivida

16 Esto afecta a toda investigación, aun cuando es evidente en las Ciencias Sociales porque se trabaja directamente con las subjetividades de los seres humanos. En las ciencias "duras" -nombre extraño y curiosopareciera que la subjetividad del investigador no perturba; sin embargo, la evidencia al respecto es contundente. Si bien la subjetividad se puede minimizar, no es posible eliminarla. 
apasionadamente desde las preguntas, antes que desde las respuestas.

\section{6. ¿Qué hacer para formar al educador investigador!}

El proceso de formación de un educador investigador puede partir de cualquier asunto, a condición que sea simple, puesto que lo simple es expresión paradojal de lo complejo; por ejemplo, ninguna investigación puede evitar la observación.

\subsection{Observar}

Observar no es más que mirar, oler, palpar, saborear, etc. con atención. Evitar la distracción es difícil y requiere mucho tiempo de práctica ${ }^{17}$. Quien no quiera dedicarle tiempo no aprenderá a observar, aunque pueda explicar en qué consiste. Los niños son buenos ejemplos de observadores atentos y concentrados, además de recordarnos cuán fácilmente se puede perder dicha capacidad. Se puede observar el vuelo de una mosca, el susurro del viento, los movimientos de los alumnos cuando están distraídos, la invisibilidad de algunos estudiantes, el uso de la pizarra, etc. Lo importante es que la observación sea dinámica y no se quede atrapada en la apariencia a que nos acostumbra la rutina. Se trata de observar lo extraordinario en lo ordinario. Observar sin pretender explicar; simplemente la persona se encuentra mirando, escuchando, palpando, tal como lo hace un niño al jugar en que se fija en todo, pero sin detenerse en nada específico. Observa sin pretender encontrar nada específico; lentamente emergerán regularidades de las que tomará nota. Nada más. Una buena observación, sostenida y relajada, además de entretener, sorprende por las regularidades que manifiesta y que se hallaban ocultas. Después el educador investigador podrá orientar la observación hacia aquellos aspectos que le interesen.

\subsection{Describir}

Para describir debe ordenar los datos distinguiendo entre la información relevante de la irrelevante, lo que podrá variar en función del sentido e intención de la descripción. Para hacerlo requerirá de vocabulario especializado y preciso, que le llevará paulatinamente a

17 Quienes meditan practicando yoga saben lo difícil que resulta mantener la atención no forzada en la propia respiración o en la llama de una vela, sin dejar que otros pensamientos perturben y, si lo hacen, dejar que se vayan sin alterar la meditación. Meditar no es pensar en "algo" sino dejarse fluir en la respiración o la vela. 
evitar la verborrea y el diletantismo; cada vez será más breve y claro en sus descripciones, al tiempo que corroborará que describir es una manera de interpretar. A medida que practique descubrirá los supuestos epistemológicos que guían sus observaciones y razonamientos. Evidentemente que esto es difícil y requiere mucha práctica, que no es mera repetición.

\subsection{Comparar}

Para comparar debe contrastar descripciones en función de criterios de mayor complejidad que le permitan hacer paralelos entre dos o más objetos. Comparar le permite jugar con los criterios: "qué pasaría si ..." y experimentar. Lo importante es que lo tome en serio, tal como cuando un niño juega. Igualmente puede suponer analogías y ver qué pasa con ellas al ponerlas a prueba. Lo más probable es que la mayoría no sean significativas ni soporten el análisis, pero eso no es importante en la fase de formación, sino la práctica que no es simple repetición.

\subsection{Analizar}

El análisis descompone el todo en sus partes. No es difícil si se trata de separar los ingredientes gruesos de una comida, pero será muy complejo cuando halla que discriminar entre sus sabores, pues no todos corresponderán al sabor original de cada ingrediente, sino al efecto sinérgico de las reacciones químicas entre ellos, lo que genera sabores peculiares y únicos ${ }^{18}$. Para ser un buen analista hay que educar la sensibilidad artística tanto como fortalecer los aspectos epistemológicos, metodológicos y técnicos necesarios. El analista siempre corre el riesgo de no poder integrarlos nuevamente si ha perdido el sentido de la totalidad. Además, que nada garantiza que el conocimiento de las especificidades permita la comprensión de la totalidad ${ }^{19}$.

\subsection{Sintetizar}

18 Esto explica por qué dos platos de comida confeccionados con la misma receta saben diferente. Las reacciones químicas de los alimentos al cocinarse crean productos diferentes, que puede apreciar aquel que degusta o saborea los alimentos, no el que los traga. Es interesante la analogía con la sabiduría que le permite al sabio "saborear" el saber y no limitarse al uso instrumental.

19 Las características del agua $-\mathrm{H}_{2} \mathrm{O}$ - no se pueden inferir de las propiedades del hidrógeno y del oxígeno, sino que emergen de la sinergia entre ellas. 
Al sintetizar se recompone el todo a partir de las partes analizadas, dejando solo las características valoradas como indispensable. La síntesis no es igual a la totalidad de lo investigado, pero lo representa a un alto nivel de abstracción. La síntesis no es la yuxtaposición de las partes, como en un crucigrama, ni un resumen, sino información algorítmica, gracias a la cual se podrá reconstruir el todo. Una fórmula científica es un buen ejemplo, al igual que una imagen holográfica.

\subsection{Simplificar la complejidad y complejizar lo simple}

El listado de estas actividades puede ser largo, pero no es necesario hacerlo en este artículo. Sin embargo, es indispensable enfatizar que el educador investigador, investigue lo que investigue, deberá clasificar, codificar, descodificar, hipotetizar, comprobar, etc. En cada caso, deberá guiarse por el criterio más simple entre todos los posibles, al modo como el agua baja la montaña buscando el terreno más bajo, gracias a lo cual la investigación no le resultará un asunto complicado, sino profundamente complejo $^{20}$.

Finalmente, hay que reiterar que el educador investigador deber tener una rigurosa y consistente formación teórica que le permita concebir su trabajo con claridad y precisión, lo que implica conocer a fondo la literatura especializada para saber qué se ha investigado y cómo se ha hecho, y evaluar el sentido y alcance de las concepciones, metodologías y técnicas de investigación en boga, gracias a lo cual estará en condiciones idóneas para diseñar una buena docencia e investigación, aunque nada le liberará del riesgo de equivocarse, que le acompañará siempre. Nada de esto es extraordinario, pues ninguna de estas exigencias es ajena al buen desempeño profesional.

\section{Referencias bibliográficas}

Atwell, B. (2015). El advenimiento de la noosfera. En F. Prima (Ed.), Nuevos Paradigmas. Entrevistas. (pp. 307-323). Málaga: Editorial Sirio.

\footnotetext{
20 Es complicado cuando no se tiene buenos criterios para iniciar la investigación; en cambio, es compleja cuando se cuenta con los criterios, aunque después resulten erróneos.
} 
Brockman. M. (ed). (2010). La ciencia del futuro. Barcelona: RBA Ediciones.

Calvo, C. (2016). Del mapa escolar al territorio educativo: disoñando la escuela desde la educación. La Serena: Universidad de La Serena.

Calvo, C. (2015). La propensión a aprender entrampada por la escolarización. Infancia, Educación y Aprendizaje, 1, 1, 22-44.

Calvo, C. (2015). La sutileza como germen educacional copernicano. Educación Física y Deportes, 24, 2, 86-112.

Calvo, C. (2012). La formación del profesor como investigador. Temas de Educación, 9, $7-11$

Gleick, James. 1988. Caos. La creación de una ciencia. Barcelona: Seix Barral.

Kaku, Michio. 2012. La física del futuro. Cómo la ciencia determinará el destino de la humanidad y nuestra vida cotidiana en el siglo XXI. Barcelona: Debolsillo

Kaku, Michio. 2014. El futuro de nuestra mente. El reto científico para entender, mejorar y fortalecer nuestra mente. Barcelona: Debate.

Laughlin. Robert B. 2010. Crímenes de la razón. El fin de la mentalidad científica. Madrid. Katz Editores.

Moraes, Maria Cândida con Juan Miguel Batalloso Navas (colab). 2015. Transdisciplinaridade, criatividades e educaçâo. Fondamentos ontológicos e epistemológicos. Sâo Paulo: Papirus Editora.

Morín, Edgard. 2016. Enseñar a vivir. Manifiesto para cambiar la educación. Barcelona: Paidós.

Punset, Eduardo. 2004. Cara a cara con la vida, la mente y el Universo. Conversaciones con los grandes científicos de nuestro tiempo. Barcelona: Ediciones Destino.

Rodríguez, Vanessa con Michelle Fitzpatrick. 2014. The teaching brain. New York: The New Press

Taleb, Nassim Nicholas. 2009. El cisne negro. El impacto de lo altamente improbable. 


\section{Buenos Aires: Paidós}

The Guardian, Tuesday 24 April 2012 17.45 BST, leído el 29 julio 2016

https://www.theguardian.com/science/2012/apr/24/harvard-university-journalpublishers-prices

Thompson, A. S. (1859), The Story of New Zealand: Past and Present, Savage and Civílized, John Murray, Londres.Vol. 1, pág. 81.]” (19)

Tuhiwai Smith, Linda. 2016. A descolonizar las metodologías. Investigación y pueblos indígenas. LOM

Wilson, Edward O. 2014. Cartas a un joven científico. Santiago de Chile: Debate 